\title{
Mesoporous gold sponge
}

\author{
Michael B. Cortie ${ }^{\mathrm{A}, \mathrm{B}}$, Abbas I. Maaroof ${ }^{\mathrm{A}}$, Nicholas Stokes ${ }^{\mathrm{A}}$ and Alessia Mortari ${ }^{\mathrm{A}}$ \\ A. Institute for Nanoscale Technology, University of Technology Sydney, \\ Broadway NSW 2007, Australia \\ B. To whom correspondence should be addressed.
}

\begin{abstract}
Mesoporous gold sponge may be prepared by the removal of aluminium from $\mathrm{AuAl}_{2}$ by an alkaline leach. The resulting material has nanoscale pores and channels, with a high specific surface area that can be exploited in electrochemical applications. For example, the material could conceivably serve as the basis of a more sensitive capacitive sensor or biosensor, as an electrode material for a high efficiency ultracapacitor, as the semi-transparent current collector in a dye sensitised photovoltaic cell, or as the lithium-storage electrode in a lithium ion cell. The properties of the sponge may be controlled by varying its density, pore size, and pore size distribution, factors which are in turn controlled by the microstructure of the precursor compound and the conditions of deposition.
\end{abstract}

Meso-, micro- and nano-porous materials of various kinds are interesting, not least because they may possess catalytic properties and/or the ability to sequestrate or filter material in a size-selective fashion. However, metals are not generally readily prepared in this form since the associated high specific surface area of metallic sponges predisposes them to rapid oxidation which is often pyrophoric or, as for example in the case of 'fulminating ruthenium', explosive. In this short communication we take the opportunity to introduce the reader to mesoporous gold, one of the few mesoporous metal sponges that is relatively stable under ambient atmospheric conditions. Aspects of the work described have already appeared recently in more specialised journal articles ${ }^{1-4}$ and the purpose of the present paper (a version of which was presented at the recent ICONN conference in Brisbane ${ }^{5}$ ) is to present only the essential aspects of mesoporous gold to readers, with the objective of encouraging wider exploitation of this interesting material. It should also be read in the context of the recent resurgence of interest in new industrial applications for gold, e.g. ${ }^{6-8}$. These new possibilities are often motivated by the insight that nanoscale gold structures are not as inert as bulk gold, and they manifest several types of useful chemical or physical surface phenomena, including catalysis.

In the present short communication we will consider only the possible electrochemical applications for this material; however, the optical properties of mesoporous gold are quite interesting too, particularly in respect of their flat spectral characteristics, ${ }^{2,9}$ and the sponge is also an active catalyst for carbon monoxide and $\mathrm{NO}_{\mathrm{x}}$ oxidation; ${ }^{10,11}$

Although mesoporous gold has been previously prepared by the de-alloying of the more active element from $\mathrm{Au}-\mathrm{Cu}$ or $\mathrm{Au}-\mathrm{Ag}$ alloys, ${ }^{12,13}$ it is probable that the selective removal of $\mathrm{Al}$ from the intermetallic compound $\mathrm{AuAl}_{2}$ ('purple gold') is a better route. $^{1,9,10,14}$ This is because de-alloying of the Al by an aqueous solution of $\mathrm{NaOH}$ or $\mathrm{KOH}$ is rapid, and the stoichiometry of the precursor, in combination with the low density of Al, ensures a large volume fraction of pores. The precursor compound itself can be prepared in bulk form by melting together of the elements ${ }^{1}$ or, as in the present work, by co-deposition of the elements as a thin film by physical vapour deposition (see Experimental section). The resulting spongy mass is generally penetrated by a high density of irregularlyshaped pores of between 10 and $50 \mathrm{~nm}$ diameter. In its pure form the $\mathrm{Au}$ sponge is not completely stable at room temperature and, if dry, it slowly sinters with an attendant reduction in porosity, changing in colour from black to yellow as it does so. Sintering of the $\mathrm{Au}$ sponge can be retarded or even prevented by the presence of residual $\mathrm{Al}_{2} \mathrm{O}_{3}$ oxide impurities on its surface and by moisture.

The spongy nature of the mesoporous gold coatings prepared in the present work is readily apparent in Figs. 1 and 2 , which respectively show a top-view and a cross-section through a coating of this material. In this case no RF bias was applied, a condition that we have found tends to generate a relatively monodisperse distribution of pore sizes. Later we will show how a bimodal distribution of pores can be obtained, if desired. XPS measurements indicate that the surfaces are of pure $\mathrm{Au}$ with negligible impurities besides Al present. BET surface areas of up to $20 \mathrm{~m}^{2} / \mathrm{g}$ have been recorded for mesoporous $\mathrm{Au},{ }^{10}$ but the increases in electrochemical capacitance are more modest, being of the order of to 3 to 10 times that of ordinary gold coatings of the same nominal surface area. ${ }^{3}$ Evidently, many of the pores in the gold sponge are inaccessible to ions during electrochemical measurements. 


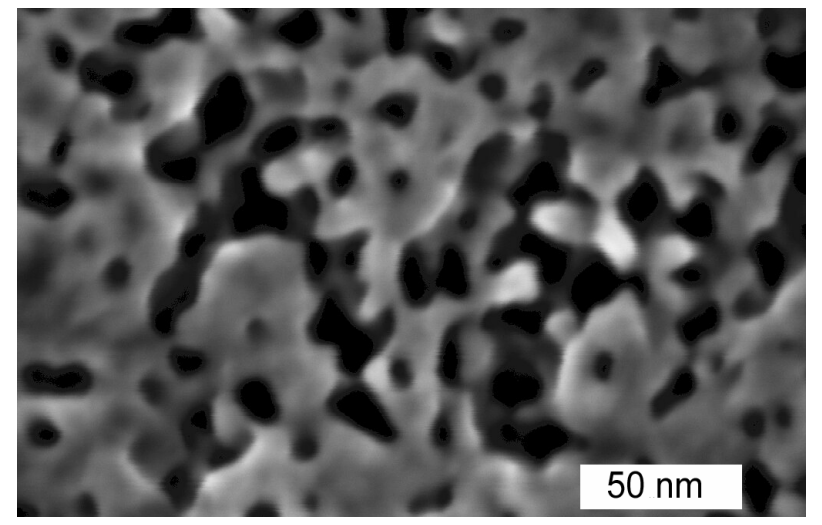

Fig. 1. Example of mesoporous $\mathrm{Au}$ sponge produced by de-alloying $\mathrm{AuAl}_{2}$ with $\mathrm{NaOH}$. The pores are of the order of $15 \mathrm{~nm}$ diameter.

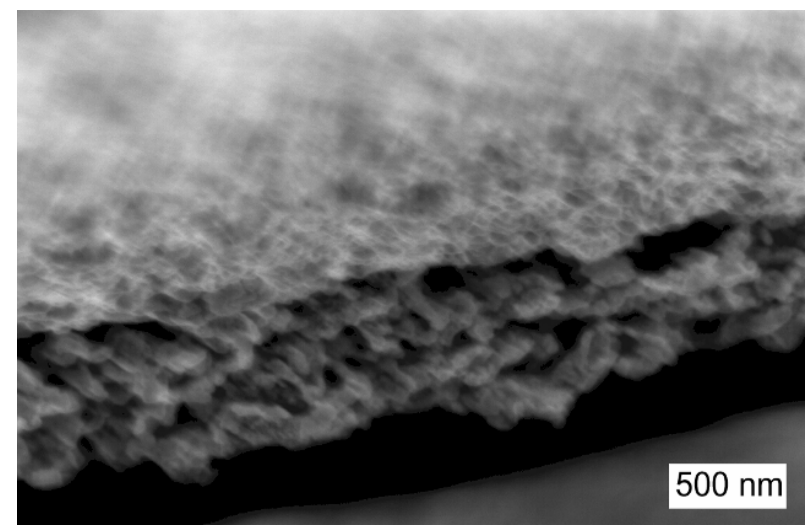

Fig. 2. Cross-section of a mesoporous gold coating produced by fracturing a coating developed on a brittle substrate, reproduced from Cortie, ${ }^{5}$ (C) 2006 IEEE.

The increased surface area of this material suggests that it could be exploited as an alternative to planar gold electrodes in electrochemical applications in which a higher specific surface area is required. Although the chemical properties of the surface of the sponge are not necessarily different from planar gold, use of a mesoporous electrode offers the prospect of electrodes of smaller overall dimensions for a given active surface area. Gold itself is an attractive material for the electrodes of many systems in any case, due to its surface chemistry which makes it a convenient platform for the attachment of proteins and other biological molecules.

The conceivable applications encompass electrochemical sensors or biosensors, whether based on capacitive or faradaic principles, high efficiency ultracapacitors, semi-transparent current collectors for dye solar cells and, perhaps, electrodes for lithium ion cells. Each of these will be briefly discussed.

Use in respect of a sensor based on faradaic principles simply exploits the greater current that would be passed by a mesoporous electrode compared to a planar one of the same macroscopic dimensions. Similarly, in the case of capacitive sensors, the double layer capacitance of such an electrode is obviously increased in direct proportion to the increase in surface area. A useful implication is that changes in current or capacitance would become more readily measurable with any particular instrumentation, particularly if based on the potential step method. This is because a higher capacitance prolongs the discharge, permitting more data to be collected in the allimportant first few milliseconds of the discharge. This is shown in Fig. 3, in which the decay transient of a $\sim 60 \mathrm{mV}$ square pulse is plotted for an ordinary planar electrode and a mesoporous one. More details of the particular experiment are available elsewhere. ${ }^{3}$ The bottom line, however, is that the decay in voltage is slower due to the greater capacitance of the system, and this provides a means to secure a more accurate transduction of the signal.

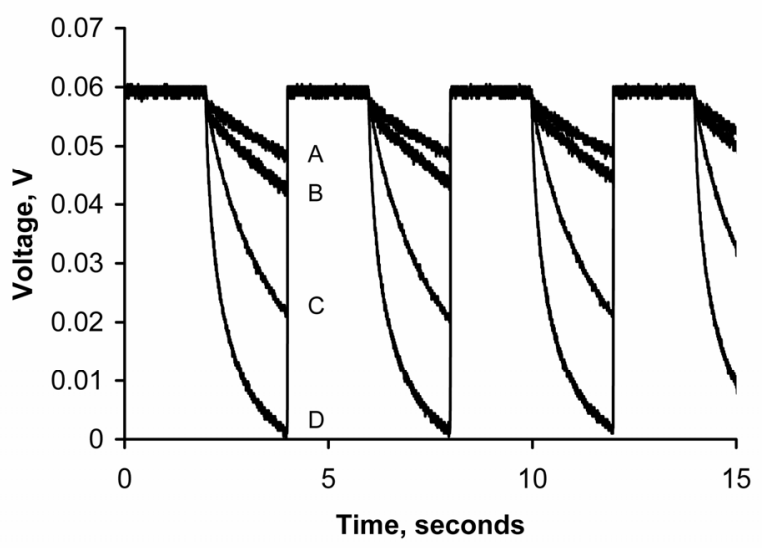

Fig. 3. Potential step experiments in physiological buffer solution, showing how increased capacitance of porous gold electrode causes slower decay of voltage. Data: A. mesoporous gold, B. mesoprorous gold with adsorbed layer of 4-mercaptophenylacetic acid (4MPAA), C. planar gold, D. planar gold with layer of 4MPAA.

A second possibility for this material may be in specialised ultracapacitors. Gold is a high value material of course, especially in comparison to the porous carbon currently favoured for these devices. Therefore, such an ultracapacitive storage device would only be useful for one reason: its lower internal resistance. While the energy, $U_{\mathrm{e}}$, that can be stored in a capacitor is given by

$$
U_{e}=\frac{C \cdot \Delta V^{2}}{2}
$$

where $C$ is the ostensible capacitance and $\Delta V$ the voltage across the device, the peak power, $P$, that can be drawn from it while discharging from voltage $V_{0}$ to $1 / 2 V_{0}$ is given by: ${ }^{15}$

$$
P=\frac{9}{16}\left(1-E_{f}\right) \frac{V_{0}^{2}}{R}
$$

where $E_{\mathrm{f}}$ is the target discharge efficiency (i.e. if $E_{\mathrm{f}}=0.8$ then 0.2 or $20 \%$ of the energy is dissipated as heat) and $R$ is the internal resistance. The power that can be supplied by the ultracapacitor at some desired efficiency is inversely proportional to its internal resistance. Halving this latter figure will double the available power (at a given threshold of electrical efficiency). So, all else being equal, an ultracapacitor based on mesoporous gold electrodes would be considerably more efficient than a conventional one over a sequence of high-power, charge- 
discharge cycles. Correspondingly, there would be no advantage whatsoever to using gold if device efficiency was an unimportant attribute.

A prototype ultracapacitor device produced at UTS is shown in Fig. 4. This device used an aqueous electrolyte, which breaks down when the potential between the electrodes exceeds about $1.5 \mathrm{~V}$. In this case no more than $\sim 2.3 \mathrm{~mJ}$ can be stored on the device shown. However, if an organic electrolyte with an operating voltage of up to $3 \mathrm{~V}$ were to be used, then it ought to be possible to store $9 \mathrm{~mJ}$ due to the quadratic dependence of $U_{\mathrm{e}}$ on $\Delta V$. Further details are available elsewhere. ${ }^{1}$

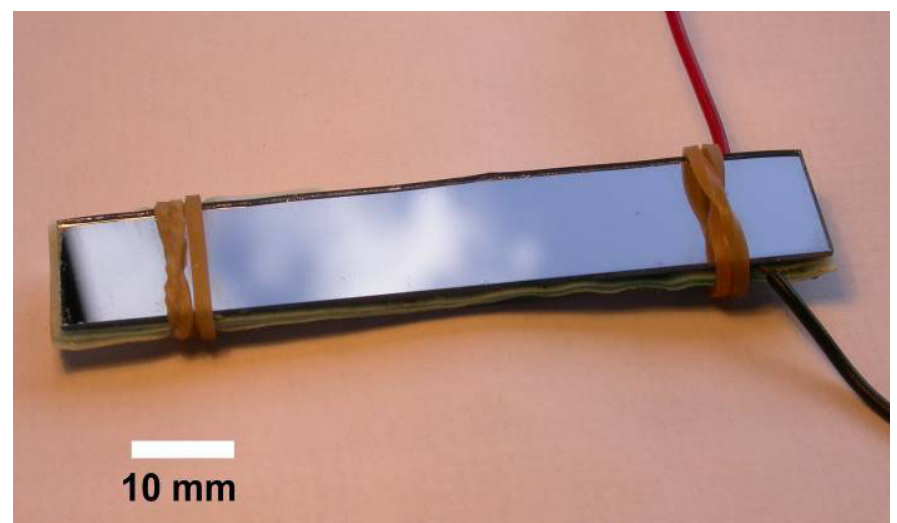

Fig. 4. Proof-of-concept ultracapacitor of $1 \mathrm{mF}$ capacity based on mesoporous gold electrodes, reproduced from Cortie, ${ }^{5}$ (C) 2006 IEEE.

The semi-transparent nature of mesoporous gold films might also turn out to be of value as transparent electrodes in dye solar cells or other electrochemical devices that convert light to energy. Although ultra-thin coatings of ordinary gold also transmit light, being particularly transparent in the range 500 to $900 \mathrm{~nm}$ (corresponding rather conveniently to the maximum in the solar spectrum), mesoporous gold coatings bring the additional advantage of being permeable to reactants as well.

Batteries might represent another application for this material. Together with colleagues at the University of Wollongong, we have shown recently that mesoporous gold electrodes can reversibly alloy and de-alloy with lithium under electrochemical control. ${ }^{4}$ These trials were conducted using an organic electrolyte and revealed that the processes of lithium insertion into the gold occurred in the voltage range of 0 to $0.25 \mathrm{~V}$ ( $v s$. a pure Li electrode), while de-alloying occurred in two stages at around $\sim 0.15$ and $\sim 0.45 \mathrm{~V}$. These potentials are a couple of hundred millivolts lower than those recorded for most other electrode materials and suggest that a lithium ion cell based on gold electrodes would have a working voltage that is greater by the same amount. Once again, this might be of value in some applications. The high surface area of the mesoporous gold enhances the possible benefit further. Cells with the mesoporous gold electrodes retained a capacity of about $80 \mathrm{~mA} . \mathrm{h}^{-1} \mathrm{~g}^{-1}$ after 30 cycles, whereas those with planar gold electrodes were only about $10 \mathrm{~mA} \cdot \mathrm{h} \cdot \mathrm{g}^{-1}$. No doubt further optimization of these preliminary results is possible, but of course the small improvements to be offered by a gold-based cell would have to be set off against its increased cost!

Finally, the range of morphologies available in this material may be expanded further by deliberately developing a bimodal distribution of porosity. There are two ways to achieve this. In the first the phase separation possibilities inherent in the Au-Al binary phase diagram are exploited. If co-deposition of the $\mathrm{Al}$ and $\mathrm{Au}$ is carried out in the two-phase region bounded by the $\mathrm{AuAl}_{2}$ and $\mathrm{Al}$ phase fields, at a temperature sufficiently elevated to allow significant diffusion to occur, then phase separation of the deposit into $\mathrm{AuAl}_{2}$ domains surrounded by a semi-continuous matrix of $\mathrm{Al}$ occurs. This segregation is readily evident in high resolution back-scatter scanning electron microscope images of appropriate precursor films, Fig. 5 , since contrast in this mode is sensitive to average atomic number. The result after de-alloying such structures is a bimodal distribution of pores, with the former $\mathrm{AuAl}_{2}$ crystallites transformed into roughly equiaxed regions of gold sponge, while the former Al-rich regions are completely removed to produce a network of open channels. Alternatively, we have also observed that application of a RF bias during deposition of the coating also induces a bimodal pore distribution, Fig 6.

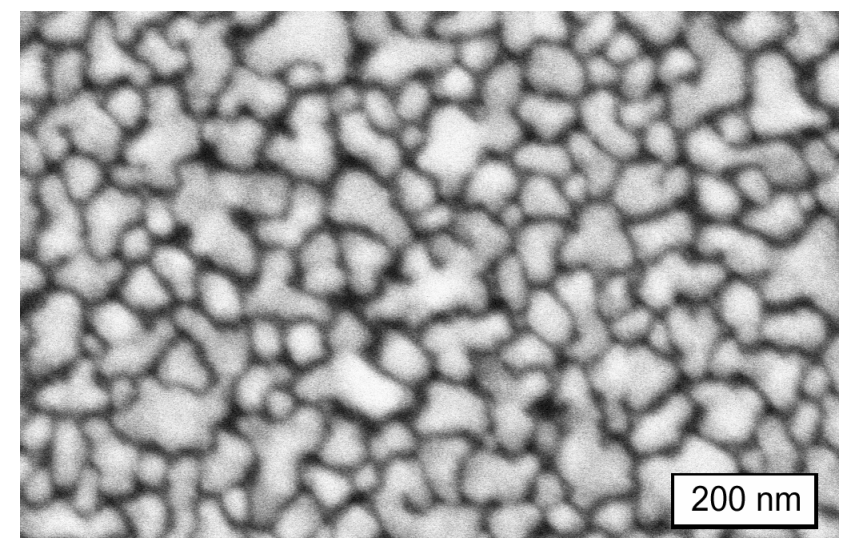

Fig. 5. Top view of precursor film viewed using electron back-scatter detection in a scanning electron microscope, showing a two-phase microstructure of $\mathrm{AuAl}_{2}$ (light) and $\mathrm{Al}$ (dark) produced when co-depositing at $400^{\circ} \mathrm{C}$, reporduced from Cortie,,$^{5}$ C 2006 IEEE.

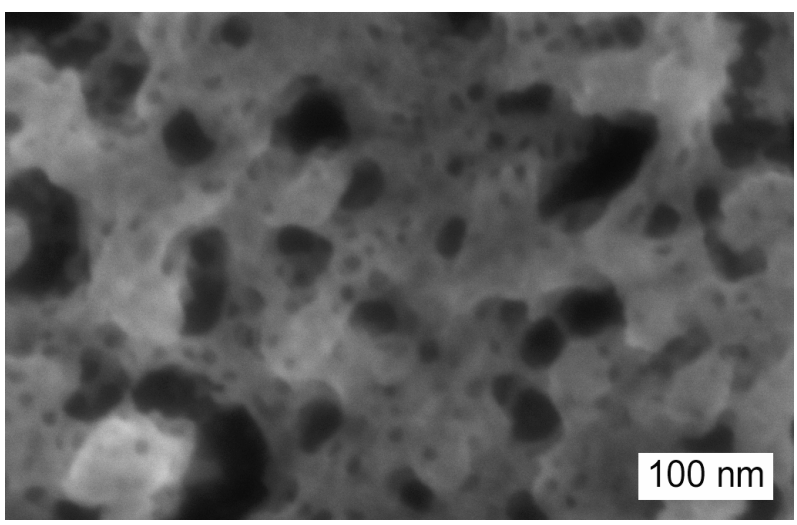

Fig. 6. Bimodal distribution of porosity in sponge produced by dealloying microstructure similar to that shown in preceding Figure. 
In summary, mesoporous gold has a significantly increased surface area compared to ordinary gold, and this can be exploited to give an increased electrochemical capacitance. Electrochemical sensors based on this material can exhibit an enhanced accuracy, particularly if they are based on the potential step concept. The morphology of the porosity can be modified by control of the microstructure, and a bimodal distribution of pore sizes can be developed if desired. We encourage the interested reader to consider this material for these and other new applications.

\section{Acknowledgements}

The authors thank the World Gold Council and University of Technology Sydney for financial support.

\section{Experimental}

Mesoporous gold films were made by de-alloying thin films comprised of a fine-scale mixture of $\mathrm{AuAl}_{2}$ and $\mathrm{Al}$, and were compared to conventional gold films. The starting coatings were prepared by deposition or co-deposition of the elements using high vacuum, direct current magnetron sputtering. The sputtering targets of $\mathrm{Au}$ and $\mathrm{Al}$ were placed $150 \mathrm{~mm}$ away from the substrate. The base pressure was lower than $\sim 1 \times 10^{-4}$ $\mathrm{Pa}$ while sputtering was carried out using a $350 \mathrm{~V}$ dc voltage in the presence of flowing $\mathrm{Ar}$ at a pressure of $0.25 \mathrm{~Pa}$. A $3 \mathrm{kV}$ $10 \mathrm{~W}$ radio frequency (RF) bias was applied to some of the substrates to provide ion etching. Samples produced with prior ion etching were far more adhesive on the glass; without ion etching the mesoporous gold was readily detached unless anchored with an underlayer of chromium or titanium. To ensure good homogeneity and crystallinity, the substrates in these experiments were heated to $400^{\circ} \mathrm{C}$ during deposition.

After deposition, the aluminium was removed by immersing the coatings in $0.2 \mathrm{M}$ aqueous $\mathrm{NaOH}$ solution for about one minute. The resulting films were neutral in colour on account of their broad and absorptive spectral characteristics ${ }^{2}$. De-alloyed samples were stored in Millipore water and used or characterised within two days. Some electrodes were subsequently immersed in an ethanolic solution of the thiol 4mercaptophenylacetic acid, a molecule known to adsorb onto gold surfaces and thereby reduce their capacitance.

\section{References}

1. Cortie, M. B., Maaroof, A. I. \& Smith, G. B., Gold Bulletin, 2005, 38, 15-23.

2. Maaroof, A. I., Cortie, M. B. \& Smith, G. B., Journal of Applied Optics A: Pure and Applied Optics, 2005, 7, 303-309.

3. Mortari, A., Maaroof, A., Martin, D. \& Cortie, M. B., Sensors and Actuators B, 2006, in press.
4. Yuan, L. et al., J. of New Materials for Electrochemical Systems, in press.

5. Cortie, M. B., Mortari, A. \& Maaroof, A. I. in 2006 International Conference on Nanoscience and Nanotechnology Proceedings (IEEE Publishing Co, Brisbane, Australia, 2006).

6. Haruta, M., Gold Bulletin, 2004, 37, 27-36.

7. Hashmi, A. S. K. \& Hutchings, G. J., Angew. Chem. Int. Ed., 2006, 45, 7896-7936.

8. Corti, C. W., Holliday, R. J. \& Thompson, D. T., Gold Bulletin, 2002, 35, 111-117.

9. Cortie, M. B., Maaroof, A., Smith, G. B. \& Ngoepe, P., Current Applied Physics, 2006, 6, 440-443.

10. Glaner, L., van der Lingen, E. \& Cortie, M. B. (ed. Mintek) (Australian Patent, 2003).

11. Cortie, M. B., Lingen, E. v. d. \& Pattrick, G. in Proceedings of the Asia Pacific Nanotechnology Forum 2003 (ed. Schulte, J.) 79-82 (World Scientific, Singapore, Cairns, Australia, 2003).

12. Erlebacher, J., Aziz, M. J., Karma, A., Dimitrov, N. \& Sieradzki, K., Nature, 2001, 410, 450-435.

13. Ding, Y. \& Erlebacher, J., Journal of American Chemistry Society, 2003, 125, 7772-7773.

14. Candy, J. P., Fouilloux, P., Keddam, M. \& Takenouti, H., Electrochimica Acta, 1981, 26, 1029-1034.

15. Burke, A., Journal of Power Souces, 2000, 91, 37-50. 